\title{
Inferring student learning style from the way they interact with a Computer User Interface and the WWW.
}

\begin{tabular}{|c|c|}
\hline Journal: & Transactions on Education \\
\hline Manuscript ID: & TE-2009-000100.R1 \\
\hline Manuscript Type: & Regular \\
\hline \multicolumn{2}{|l|}{$\begin{array}{r}\text { Date Submitted by the } \\
\text { Author: }\end{array}$} \\
\hline \multirow[t]{2}{*}{ Complete List of Authors: } & $\begin{array}{l}\text { Sanders, David; University of Portsmouth, Faculty of Technology } \\
\text { Bergasa-Suso, Jorge; Counterpoint MTC, R \& D }\end{array}$ \\
\hline & $\begin{array}{l}\text { User modeling, Data models, Learning systems, User interfaces, } \\
\text { User interface human factors, Human factors, Unsupervised } \\
\text { learning, Social factors, Engineering education, Computer } \\
\text { peripherals, Computer interface human factors, Military information } \\
\text { systems, Information technology, Military data processing, } \\
\text { Knowledge based systems, Decision support systems, Internet, } \\
\text { Data processing, Intelligent tutoring systems, Interactive } \\
\text { computing }\end{array}$ \\
\hline
\end{tabular}

\section{^scholarONE" \\ Manuscript Central}




\title{
Inferring student learning style from the way they interact with a Computer User Interface and the WWW.
}

\author{
David A Sanders and Jorge Bergasa-Suso
}

\author{
Corresponding author: $\quad$ Dr David Sanders \\ Faculty of Technology \\ University of Portsmouth \\ Portsmouth \\ P01 3DJ United Kingdom
}




\begin{abstract}
Recent significant advances in automatically predicting user learning styles are described. The system works with new client-based systems that filter Web pages and provide easy, structured, focused, and controlled access to the Internet. A first system called iLessons was embedded within Microsoft Internet Explorer 6 and provided teachers with tools to create lesson Web pages, define zones of the Internet that could be accessed during a lesson, and enforce these settings in a set of computers. A second system enabled students to investigate and collaborate using the Internet. The system filtered Web pages based on the relevance of their contents and assisted students by inferring their learning style (active or reflective) and by recommending pages found by fellow students based on page relevancy, student learning style, and state of mind measured by activity. The system infers learning style in real time by monitoring user activity and recent significant advances in the research are described. [154 words]
\end{abstract}

Keywords: Cooperative/collaborative learning, distributed learning, intelligent tutoring systems, navigation. 


\section{Inferring student learning style from the way they interact with a Computer User Interface and the WWW.}

\section{INTRODUCTION}

A system was first described in this journal that assessed student learning style and activity from the way that they interacted with their User Interface [1]. Results were then used to recommend suitable pages found by other students with a similar learning style. Experiments in [1] found some initial rules about user activity and in the characteristics of Web pages that could predict some student learning styles based on their behaviour while browsing the Internet.

That work in [1] and the new work described in this paper were tested with groups of University students who had different learning styles while they performed various engineering research tasks on the WWW and an agent monitored their activity. Recorded data were fed into a model that returned the predicted dimensions for each user. The volunteers then completed an Index of Learning Styles (ILS) Questionnaire that defined their learning styles and the questionnaire results were compared with those predicted by the automatic models for each student.

\section{BACKGROUND}

Engineering teachers sometimes find the Internet difficult to use in a teaching environment. A lack of control over student access and systems can be complex to manage [2]. Teachers are rarely IT specialists and new tools have been required to provide structured, focused, and controlled access to the Internet. The new systems 
created during the research described in this paper overcome these difficulties and are applicable to most courses using the Internet.

\begin{abstract}
A broad range of internet education systems are in the market such as WebCT [3] but they do not provide intelligent advice on potential sites or consider student activity. These limitations suggested a need for another type of software tool [4]. Newer systems attempted to meet this need, such as iLessons, a Web browser-based system embedded within Microsoft Internet Explorer [5] that was created during the research.
\end{abstract}

A problem was that the systems lacked adaptability to meet student learning styles and did not provide any collaboration facilities. Intelligent web-based systems, such as INSPIRE [6], ARTHUR [7], AES-CS [8], LSAS [9], CS388 [10], Tangow [11], AHA!, and MOT [12] have attempted to provided intelligent content adaptation based on learning style and previous knowledge but these systems have needed to assess students' learning styles off-line and through questionnaires. iWeaver and AHA! have attempted to dynamically adapt to preferences by monitoring user feedback and navigation patterns but none of these systems use Web pages readily available on the Internet, or infer students' learning styles by analyzing the way users navigate and interact with the WWW.

Because iLessons used a Web browser-based system it was relatively easy-to-use. Focus was provided by allowing teachers to decide which Internet pages or domains students were allowed to access; structure was provided by the ability to create lesson Web pages within the browser, and to collect and reuse Internet resources by drag \& 
drop. Control was achieved by saving this information into a single lesson file that could be applied to student computers grouped by class or by task.

Client-based educational systems that do not rely on proprietary servers have the advantage that an extra network infrastructure such as a proxy server or a Web server is not needed. Files can be stored in a network file server or locally. These systems are scalable; a single client can be installed in a computer with a dial-up connection or in every computer in a network without extra overhead. A benefit of Web server-based educational systems is that they can be accessed from a standard Web browser. Reusing functionality available in commercial Web browsers such as Internet Explorer makes the client-based system easier to use and to implement. Internet Explorer's functionality can be extended by explorer bars [5], tool bands, intelligent add-os [13], Browser Helper Objects, and Asynchronous Pluggable Protocols (APPs) [14]. Extensions of this sort were used in this research to provide extended functionality within a standard Web browser.

\section{THE ILESSONS SYSTEM}

iLessons was a novel set of tools that overcame the limitations of other Web-based educational authoring tools by giving teachers the ability to reuse materials readily available on the WWW. Drag and drop was used to create a set of Web pages with Internet access restrictions, all from within a standard Web browser and without the need for server software. The iLessons user interface consisted of a toolbar and a left-side pane embedded into the Internet Explorer UI as shown in figure 1. iLessons was tested at all academic levels by lecturers, teachers, and students and was made available for testing on the WWW [1]. 


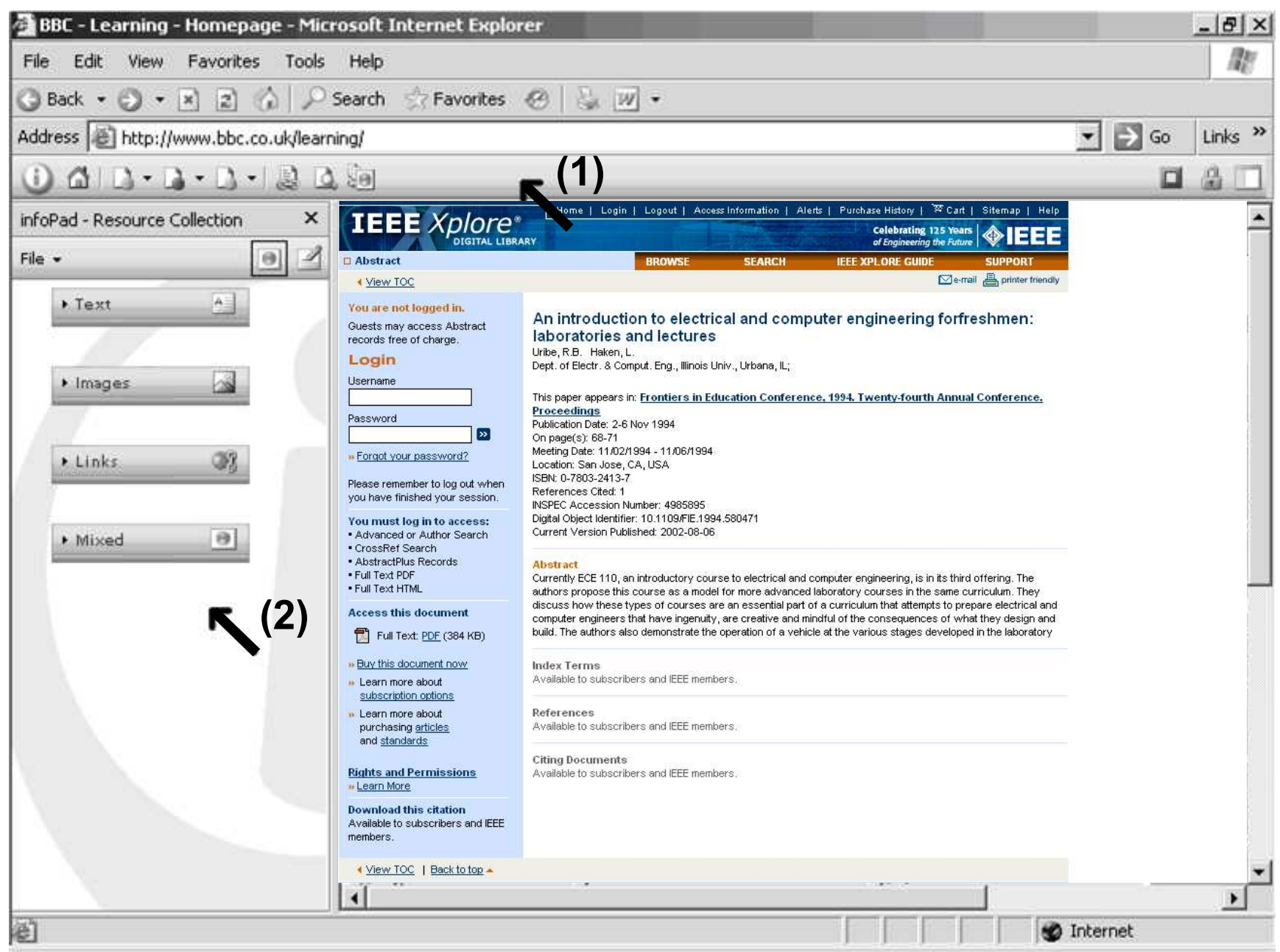

Figure 1: iLessons User Interface (UI) embedded into Internet Explorer showing (1) toolbar and (2) a left-side pane embedded into the Internet Explorer UI

Teachers could use a content editor subsystem to gather resource collections; edit lesson Web pages; allow, deny, and trust zones of the Internet; and assign a particular lesson to a classroom. Teachers could collect resources, such as plain text, images, links, and html fragments from WWW pages by navigating to them with Internet Explorer and dragging and dropping relevant parts into the iLessons left-side pane. Resources could be used later to create lesson Web pages. 
iLessons blocked access to the Internet by default in student mode, unless teachers granted free access. Teachers could also grant access to specific areas of the Internet (pages, domains, or directories) so that students had limited but focused access. Web pages, domains and directories could also be "trusted" so that when students were viewing a trusted page, they were automatically allowed to navigate to any link from the page and to any link from those pages, until the depth specified by the teacher was reached. For example, directories and domains containing relevant pages, such as www.school-for-champions.com/ ${ }^{*}$ and http://mathworld.wolfram.com/* could be allowed to grant students access to supporting material.

\begin{abstract}
Students were also able to create course work files in Word format from within Internet Explorer.
\end{abstract}

Local Educational Authority advisors from the UK Department for Education and Skills investigated and tested iLesssons and strongly supported the new systems. iLessons was asserted as an easy-to-use, novel, and effective tool to deliver guided learning using the Internet in a classroom.

Despite the positive response, it was realised that iLessons could have been more flexible and adaptable, and could have included collaboration facilities to enable students to use the Internet as a research group tool. Feedback from teachers using iLessons suggested that while allowing and denying specific areas of the Internet was an effective way of controlling the misuse of the Internet during a lesson and of focusing students', they were not able to use the Internet to carry out their own research. Research therefore moved on 
to the creation of a new intelligent system to provide a less restrictive filtering mechanism based on the relevance of WWW pages to a subject, and to consider individual student learning styles, using iLessons as a platform.

\section{A NEW INTELLIGENT SYSTEM}

A first prototype of the new intelligent system featured an intelligent document classification system. Teachers trained the system by ranking a set of Web pages with a relevancy rating. New lessons were created, but teachers provided relevancy ratings for a set of pages for each lesson as examples for the intelligent document classification system instead of allowing or denying access to pages, directories, or domains. Irrelevant pages were also rated. The system analyzed text contained within example pages and obtained a rating pattern from the training set that consisted of a weighted set of words that determined the characteristic contents of relevant pages. The rating pattern was loaded into the student system. When students requested Web pages, their contents were extracted and compared with the rating pattern, which provided a rating of the similarity between the requested page and the relevant pages. Only pages with a similarity rating above a pre-set threshold were allowed. The result was a more liberal filtering approach. Students could navigate to any page, as long as its contents were related to the relevant pages in the training set. After a seminar, students could continue researching through access to any pages related to the subject without being distracted by irrelevant content.

Subject-specific filtering using document categorization was flexible enough to enable students to use the Internet as a research tool, but the system lacked adaptability to meet 
student learning styles and did not provide any collaboration facilities. Some intelligent Web-based systems have provided intelligent content adaptation based on learning style and previous knowledge. These systems worked with Web-based adaptive material created with proprietary tools. They assessed students' learning styles through questionnaires, and iWeaver and AHA! dynamically adapted to preferences by monitoring user feedback and navigation patterns. None of these systems used Web pages readily available on the Internet, or infered students' learning styles by analyzing the way users navigated and interacted with the WWW.

The learning experience of individual students and groups using iLessons was enhanced by a collaborative agent system that recommended Web pages from the WWW to students based on three dimensions:

- page relevancy based on contents,

- page layout based on student learning style inferred by the system,

- $\quad$ and user activity (interested or inactive).

When a student requested a Web page, the page was approved only if it matched a relevance pattern. If the page was relevant, its structure was compared with a pagelearning style pattern to find the suitability of the page for each learning style. The UI agent then sent a notification to a collaborative agent that recommended the Web page to other students according to their learning styles. The learning style of each student was initially determined by questionnaire but later the systems were improved to determine learning style using a software agent that analyzed interaction with the UI. A user activity analyzer detected whether a student was participating by timing the UI. To encourage 
them to join the learning experience after periods of inactivity, students had pages suggested to them so that they could navigate to a page that suited their learning style.

In order to determine the learning style of a student automatically, patterns needed to be found in the way users with different learning styles made use of the Internet. Also necessary was finding patterns in the layout and elements of Web pages that were easier to understand by students depending on their learning style. Various models of learning style were considered [15] - [20]. The Felder-Silverman dimensions of learning style model was a well-accepted model used in several adaptive hypermedia applications such as LSAS, CS388, and Tangow. It was selected for further study before fully coding the new systems because it provided four dimensions of learning style $[18,19]$ that might be measured from data obtained from the computer systems: timings, actions, locations, etc. The dimensions were:

\section{A. Active-Reflective}

Active learners tend to retain and understand information best by doing something active, such as discussing, applying it or explaining it to others. Reflective learners prefer to think about it quietly first.

\section{B. Sensing-Intuitive}

Sensing learners tend to like learning facts; intuitive learners often prefer discovering possibilities and relationships. 


\section{Visual-Verbal}

Visual learners remember best what they see: pictures, diagrams, flow charts, time lines, films and demonstrations. Verbal learners get more out of words: written and spoken explanations. Everyone learns more when information is presented both visually and verbally, but most are visual.

\section{Sequential-Global}

Sequential learners tend to gain understanding in linear steps. Global learners tend to learn in jumps, absorbing material almost randomly and then suddenly "getting it."

\section{CREATION AND TESTING OF PATTERNS}

An experiment was conducted to find rules in $U I$ activity and in the characteristics of Web pages that would predict student learning style based on their behaviour while browsing the Internet, and the suitability of a given Web page for each learning style dimension.

An experimental system was created to record and analyze data. An agent registered UI interaction while the user was investigating a subject. User activity and page structure were analyzed and recorded each time a page was visited. User data was stored in a database tagged with the user's dimensions of learning style questionnaire results, so that it could be processed by a data mining engine along with data from other users. The parameters recorded by the agent were: time in a page; mouse speed, total mouse distance, mouse distance in $X$ and $Y$ axis; scroll speed, scroll distance, changes in scroll direction; use of back and forward buttons; data copied and data dragged. The page structure parameters recorded were: length of page text; number and area of images; 
ratio of text to images, presence, and location of tables, bulleted and numbered lists, presence of sound files, video files, animations and ActiveX components; presence and location of question marks, and keywords, such as: "example", "figure", "question" and "diagram".

A group of 20 users (with different learning styles) from the initial group volunteered to investigate a subject for 15 minutes using Internet Explorer while the agent monitored their activity, and to rate each page depending on how easy that page was to understand. Once information had been gathered from the group of users, the data was analyzed to find correlations between: dimensions of learning style, UI usage, and the way information was presented in useful pages. Correlations were found between each of the dimensions of learning style and the UI activity and page content parameters monitored by the agent. The 20 parameters monitored by the agent that were most significant to predict each dimension were extracted. As an example, the five most significant parameters to predict whether a user is Active or Reflective were:

1) $\frac{\text { Area of images }}{\text { Text length } \times \text { Scroll direction changes }}$

2) Average time spent on page

3) $\frac{\text { Area of images }}{\text { Text length } \times \text { Time spent in page }}$

4) $\frac{\text { Area of images }}{\text { Text length } \times \text { Scroll distance }}$

5) $\frac{\text { Image count }}{\text { Text length } \times \text { Mouse distance in } Y \text { axis }}$ 
These parameters and the values recorded for each user were utilized to create a probability model for each dimension of learning style that could predict the dimensions of learning style of new users based on the value of each selected parameter as recorded by the agent. This model returned a percentage of certainty that a user belonged to one of the extremes of each dimension of learning style.

To test the effectiveness of the model, a group of seven students (with different learning styles) were given the same research task as the initial group. The agent monitored their activity and the data recorded by the agent was fed into the model, which returned the predicted dimensions of each user. The students then completed ILS Questionnaires, and the questionnaire results were compared with those predicted by the models for each student.

Using a similar method in [1], the model to predict a student as active or reflective was only slightly more accurate than the naive prediction calculated from a sample population but that work was a first success even though the results were relatively naïve.

In this work, prior to the experiment, a group of 67 users completed the ILS Questionnaire [21], and the results were entered into a database. In this way, the preferred learning style of each user was known before the experiments, along with the distribution of the different dimensions of learning style over the sample population (see Table 1).

The percentage of users belonging to each dimension determined the minimum accuracy required for the rules. For example, a naive prediction that considered every user as 
Active would have an accuracy of $57 \%$ according to Table 1 . Any rule found to predict a user's dimension of learning style must be more accurate than the naive prediction for the given dimension. Results from questionnaires were not disclosed to the users until after the experiment finished.

\begin{tabular}{|l|c|c|}
\hline Dimension & $\begin{array}{c}\text { Number of } \\
\text { people }\end{array}$ & \% \\
\hline Actives & 38 & $57 \%$ \\
\hline Reflectives & 29 & $43 \%$ \\
\hline Sensors & 35 & $52 \%$ \\
\hline Intuitives & 32 & $48 \%$ \\
\hline Visuals & 51 & $76 \%$ \\
\hline Verbals & 16 & $24 \%$ \\
\hline Sequentials & 23 & $34 \%$ \\
\hline Globals & 44 & $66 \%$ \\
\hline
\end{tabular}

\section{Table 1: Distribution of dimensions of learning style over sample population}

In the intitial tests for Sensing/Intuitive, Visual/Verbal and Sequential/Global, the results were only equivalent to guessing. They are reproduced in Table 2.

\begin{tabular}{|l|c|c|}
\cline { 2 - 3 } \multicolumn{1}{c|}{} & Accuracy & $\begin{array}{c}\text { Naïve pred. } \\
\text { Accuracy }\end{array}$ \\
\hline Active/Reflective & $71 \%$ & $57 \%$ \\
\hline Sensing/Intuitive & $29 \%$ & $52 \%$ \\
\hline Visual/Verbal & $71 \%$ & $76 \%$ \\
\hline Sequential/Global & $57 \%$ & $66 \%$ \\
\hline
\end{tabular}

Table 2: Accuracy of models and required accuracy in Bergasa et al- 2005 [1].

The new methods described in this paper significantly improved on these results by introducing a new unknown set for pairs of significant parameters. 
As an example, some scatter graphs showing some results from twenty users are shown in figures 2 and 3 . Figure 2 is a three dimensional scatter graph showing user data points against three of the attributes used to classify a user as Active or Reflective:

- Amount of mouse movement in the $Y$ axis;

- Ratio of document length to time spent on page;

- and Ratio of images area to document length and scroll distance.

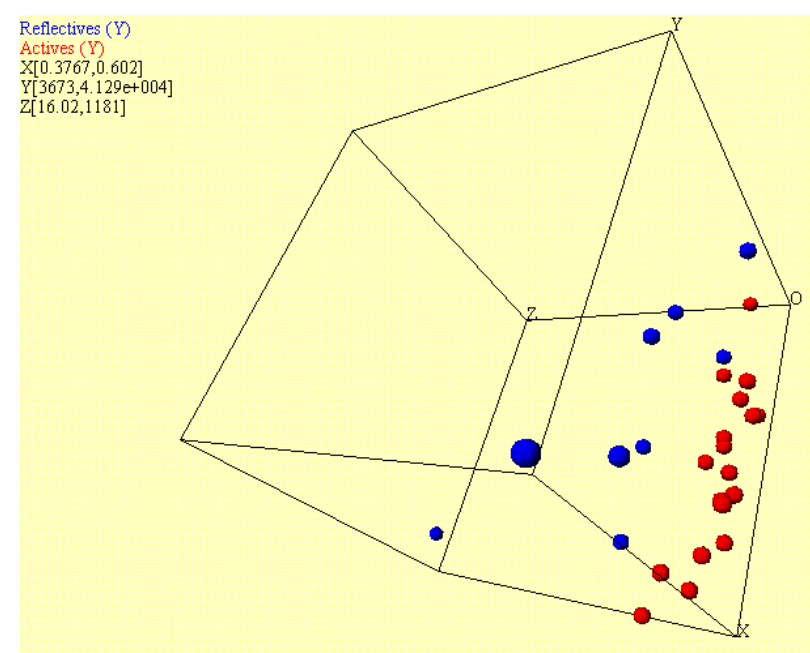

Figure 2: Clusters of Active (red) and Reflective (blue) users visualised by plotting data points against "amount of mouse movement in the $Y$ axis" (plotted on the $X$ axis), "ratio of document length to time spent on page" ( $Y$ axis); and "ratio of images area to document length and scroll distance" ( $Z$ axis).

To make the clusters easier to visualise and the method easier to describe, figure 3 shows the clusters of Active (red/diamond) and Reflective (blue/square) users in two dimensions. Data points are plotted against "Amount of mouse movement in the $Y$ axis" (X) and "ratio of images area to document length and scroll distance" $(\mathrm{Y})$. 


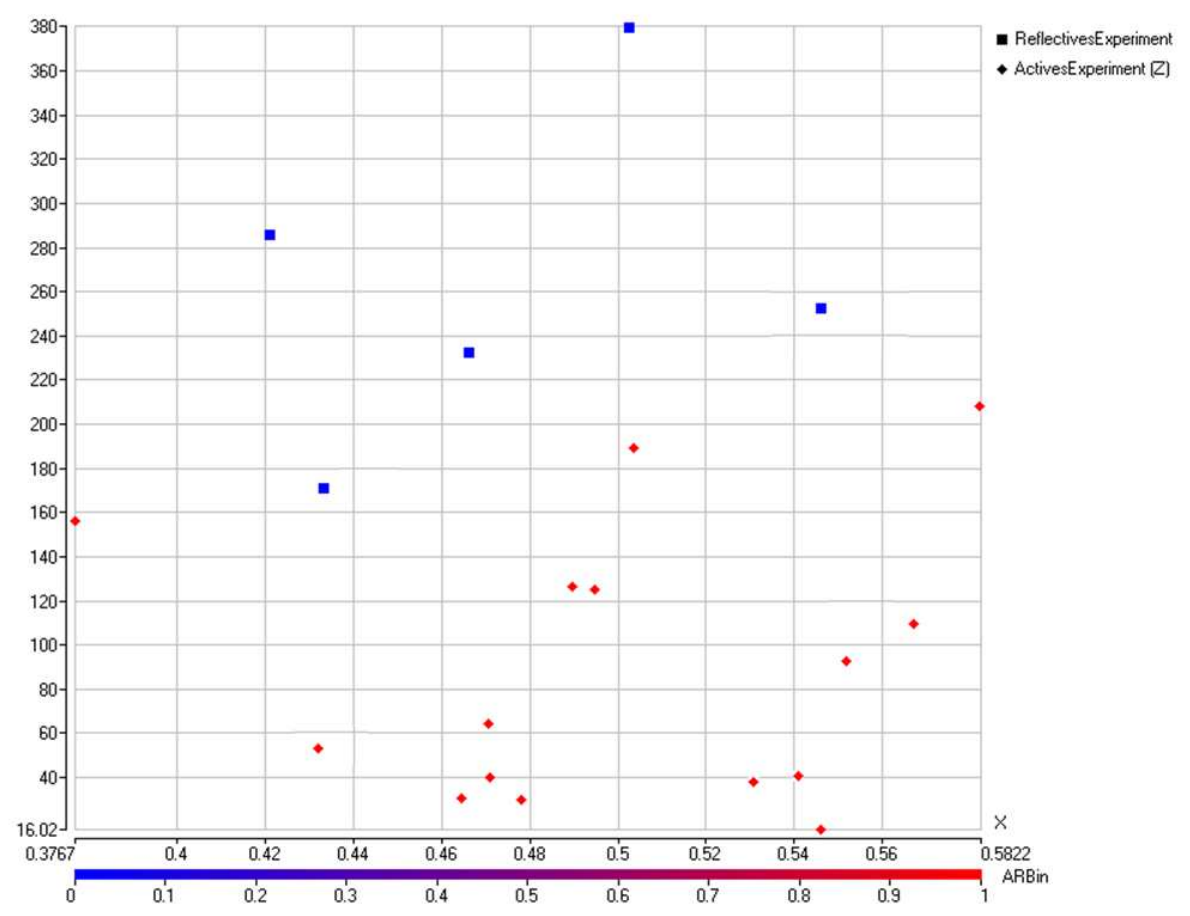

Figure 3: Clusters of Active (red/diamond) and Reflective (blue/square) users visualised by plotting data points against "Amount of mouse movement in the $Y$ axis" $(X)$ and "ratio of images area to document length and scroll distance" $(Y)$.

The clusters overlap and for example, one of the Reflective responses can be seen well over to the right hand side in figure 3 (possibly within the Active cluster) and some of the Active responses could be considered to be within the Reflective Cluster. A problem with the novel work in [1] was that users would always be considered to either belong to the set of Active Users or to belong to the set of Reflective Users in every test. Each of the twenty most useful sets of parameters made a decision about each dimension and the probability of a user being one or other dimension was then calculated from the twenty decisions. This was fine for users who clearly fell into one or other category all the time (or nearly all the time) but for most people, they only tend towards one dimension or the other (and even that might change a little depending on circumstances, mood or need). 
In this new work, each two-dimensional cluster was further defined by creating some dead bands within which a user was not defined as either dimension. This was achieved by either setting a radius during the learning process or by setting a straight line boundary, as shown in figure 4. Each radius (or line) was calculated during the learning process by considering the two-dimensional position of each of the users within each cluster of dimensions.
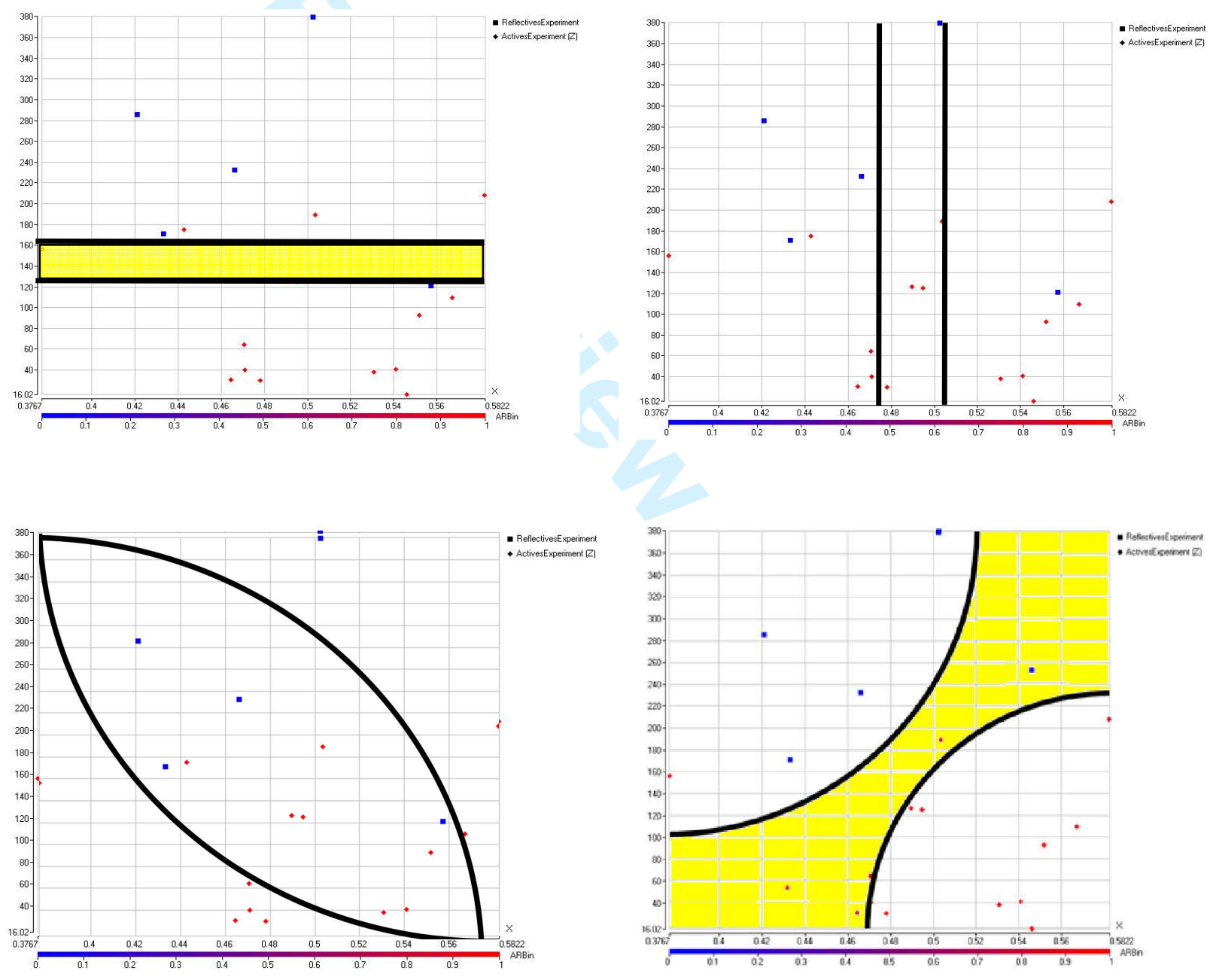

Figure 4: The two dead bands created for sets of attributes in two dimensions that could be used to classify a user as Active or Reflective or Unknown. 


\begin{abstract}
A radius line (or straight line) was then introduced so that the $80 \%$ of users with a particular dimension who were nearest to the origin (or edge) were placed into a set. The other $20 \%$ were outside the radius line (or straight line) and not recorded as being part of the set. If the two lines did not overlap, then this sometimes created a dead-band that contained users with less certain results. The graph in the top left corner of figure 4 and the graph in the bottom right hand corner of figure 4 both have dead bands shown by the shaded area. In those two cases, that introduced a new unknown set of results for each pair of useful parameters so that users were either Active, Reflective or Unknown. The graph in the bottom left corner of figure 4 and the graph in the top right hand corner of figure 4 do not have dead bands. That is because in those cases the $80 \%$ lines overlapped each other.
\end{abstract}

If there was a choice of more than one set of data with a dead band then the widest dead band would be selected. So in this case, the graph in the bottom right hand corner of figure 4 shows the shape of the sets selected for Reflective / Active dimensions using the "Amount of mouse movement in the $Y$ axis" (plotted on the $X$ axis) and "ratio of images area to document length and scroll distance" (plotted on the $Y$ axis). That was because the distance between the curved lines was greater in the graph in the bottom right hand corner than the straight lines in the graph in the top left hand corner of figure 4.

The algorithm to calculate the dead band during the learning phase is included as an Appendix at the end of this paper. 
The sets produced for the parameters shown in figure 5 were: Active, Reflective and a new set "Unknown". Users were only classified as probably Active or Reflective for each pair of parameters if they were outside the dead band and therefore clearly within one of the two sets bounded by the established lines. That effectively removed less certain cases from individual pairs of results so that when all the results were collated, the final results were more certain and less naïve.

\section{TESTING OF PATTERNS AND THE RESULTS}

A significant advance occurred when the additional programming was incorporated to add in the unknown set for each pair of sets of results from the data-mining.

This had a significant effect on three of the sets of results, those for Active/Reflective, Visual/Verbal and Sequential/Global, as shown in Table 3. Using this new method, a very small number of users were sometimes not defined by the system because they always fell into the unknown set for every pair of useful parameters (less than $2 \%$ ).

\begin{tabular}{|l|c|c|}
\cline { 2 - 3 } \multicolumn{1}{c|}{} & Accuracy & $\begin{array}{c}\text { Näive pred. } \\
\text { Accuracy }\end{array}$ \\
\hline Active/Reflective & $81 \%$ & $58 \%$ \\
\hline Sensing/Intuitive & $40 \%$ & $51 \%$ \\
\hline Visual/Verbal & $82 \%$ & $74 \%$ \\
\hline Sequential/Global & $69 \%$ & $67 \%$ \\
\hline
\end{tabular}

\section{Table 3: Accuracy achieved by the new system after dead bands were introduced and the required accuracy before results could be considered significant.}

Table 3 shows a significant improvement over the most recently published results that were reproduced in Table 2. The results for determining whether a user was Sequential 
or Global became significant for the first time. The accuracy for determining whether a user was Active or Reflective OR Visual or Verbal increased significantly. The accuracy for determining between Sensing / Intuitive remained below the best result that could be achieved by guessing but the accuracy for determining between Active / Reflective users increased from $71 \%$ to $81 \%$. In addition, the accuracy for determining between Visual / Verbal users increased from $71 \%$ to $82 \%$ and accuracy for determining between Sequential / Global increased from $57 \%$ to $69 \%$.

\section{DISCUSSION AND CONCLUSIONS}

iLessons has already been successfully used and tested in twenty three schools and colleges, but students using the system were not able to use the Internet to carry out their own research. Also, iLessons did not take into account students' learning styles or provide ways to collaborate while researching. The new intelligent system overcomes these limitations, featuring non-restrictive intelligent document filtering and consideration of student learning styles and activity.

The new systems do not seek to replace teachers, but to assist them by releasing them from monitoring the use that students make of the Internet and making them more available to enhance the learning experience. Collaborative Web page recommendations that consider the learning style of each student could be a powerful way of assisting a student in assimilating knowledge from resources readily available on the WWW. The system may improve effectiveness and efficiency of group learning by recommending pages to students with an often similar learning style (active or reflective) and to remove teacher bias. 
The introduction of a new unknown set for each pair of useful parameters allowed the creation of more accurate rules to predict the Active/Reflective, Visual/Verbal and Sequential/Global dimensions of learning style and for the first time, rules were found for the Sequential / Global dimensions of learning style.

Collaborative Web page recommendations that consider the learning style of each student could be a powerful way of assisting a student in assimilating knowledge from resources readily available on the WWW [1]. This new method appears to improve the effectiveness and efficiency of group learning by recommending pages to students with an often similar learning style (active or reflective) and to remove teacher bias... but this work is ongoing.

Research is also ongoing to consider new applications for this work but for the moment is concentrating on improving models for the fourth dimension of learning style (Sensing/Intuitive) The current work only used a keyboard, mouse and standard display screen but future research will embrace different user interfaces, including touch screens[22,23], pointer devices [24] and joysticks [25,26], and finding correlations between the relevance rating of Web pages and their usefulness for each of the learning styles. At the time of writing, some research is just beginning to investigate the effect of adjusting the threshold settings on the algorithms for calculating the different learning styles (and relevance of pages) in real time. Future work will test more users to further verify the measurements and effectiveness of the adaptation.

[4,100 words] 


\section{APPENDIX - Pseudo code for the algorithm to create the dead bands}

Count the set of D1_Users to give D1_TOTAL

Count the set of D2_Users to give D2_TOTAL

List the set of D1_Users in a set of tables (D1T1 - D1T8).

List the set of D2_Users in a set of tables (D2T1 - D2T8).

Then for each D1_user calculate distance from:

- top left corner using Pythagoras to give RADIUSTL(D1) and place in D1T1.

- bottom left corner using Pythagoras to give RADIUSBL(D1) and place in D1T2.

- top right corner using Pythagoras to give RADIUSTR(D1) and place in D1T3.

- bottom right corner using Pythagoras to give RADIUSBR(D1). and place in D1T4.

Then for each D2_user calculate distance from:

- top left corner using Pythagoras to give RADIUSTL(D2) and place in D2T1.

- bottom left corner using Pythagoras to give RADIUSBL(D2 and place in D2T2.

- top right corner using Pythagoras to give RADIUSTR(D2) and place in D2T3.

-bottom right corner using Pythagoras to give RADIUSBR(D2) and place in D2T4.

Then for each D1_user calculate distance from:

- top using $Y$ axis values to give LINET(D1) and place in D1T5.

- bottom using $Y$ axis values to give LINEB(D1) and place in D1T6.

- right side using D1 axis values to give LINER(D1) and place in D1T7..

- left side using D1 axis values to give LINEL(D1) and place in D1T8.

Then for each D2_user calculate distance from:

- top using $D 2$ axis values to give LINET(D2) and place in D2T5.

- bottom using $D 2$ axis values to give LINEB(D2) and place in D2T6.

- right side using $X$ axis values to give LINER(D2) and place in D2T7.

Then:

- left side using $X$ axis values to give LINEL(D2) and place in D2T8.

Sort XT1 to XT4 so that longest values are at the top and shortest at the bottom. Sort YT1 to YT4 so that longest values are at the top and shortest at the bottom. Sort XT5 to XT8 so that longest values are at the top and shortest at the bottom. Sort YT5 to YT8 so that longest values are at the top and shortest at the bottom.

Then:

For $n=1$ to 8 :

Find the D1T1 $-D 1 T 8$ that are $80 \%$ from the bottom $=D 1 T n(80 \%$ value $)$.

Find the D2T1 $-D 2 T 8$ that are $80 \%$ from the bottom $=D 2 T n(80 \%$ value $)$.

Then:

For $n=1$ to 4 :

"Width of dead_band", $D T n=D 1 T n(80 \%$ value $)-D 2 \operatorname{Tn}(80 \%$ value $)$.

Then

Find maximum value of DTn "Width of dead_band"

If $D T n>0$

Then

End

Select that DT and create sets. 


\section{REFERENCES}

[1] Bergasa-Suso J, Sanders DA and Tewkesbury, GE. Intelligent browser-based systems to assist Internet users. IEEE Transactions on Education 48 (4), pp 580-585. Nov 2005.

[2] Bergasa-Suso J, Sanders DA, Close A, and Tewkesbury GE, "A caught in the act filter to assist in using the Internet". In Proceedings of the 4th International Conference on Information Communication Technologies in Education, 2003, pp. 225-230.

[3] WebCT (2009) WebCT Community Resources. [Online] Available: http://www.Webct.com

[4] Sanders D (1993). System Specification 2. Microprocessing \& microprogramming 38 (1-5), p 833.

[5] Microsoft Corporation (2009) Microsoft Internet Explorer 6: Technology Overview. [Online] Available: http://www.microsoft.com/windows/ie/evaluation/overview/default.asp.

[6] K.A. Papanikolaou, M. Grigoriadou, G.D. Magoulas and H. Kornilakis, "Towards New Forms of Knowledge Communication: the Adaptive Dimension of a Web-based Learning Environment", Computers and Education, 39(4), pp. 333-360, 2002.

[7] J. E. Gilbert, C. Y. Han, “Adapting instruction in search of 'a significant difference”, Journal of Network and Computer applications, 22(3), pp. 149-160, 1999.

[8] E. Triantafillou, A. Pomportsis, and E. Georgiadou, "AES-CS: Adaptive Educational System base on cognitive styles", In Proceedings of the AH2002 Workshop, 2002, pp. 10-20.

[9] Learning Styles Adaptive System (LSAS) (2009) [Online] Available:

http://www.archives.ecs.soton.ac.uk

[10] C. A. Carver, R. A. Howard and W. D. Lane, "Enhancing student learning through hypermedia courseware and incorporation of student learning styles”, IEEE Trans on Education, 42(1), pp. 33-38, 1999.

[11] P. Paredes and P. Rodriguez, "Considering sensing-intuitive dimension to exposition-exemplification in adaptive sequencing", in Proceedings of the AH2002 Conference, 2002, pp. 556-559.

[12] N. Stash, A. Cristea, P. De Bra, "Authoring of learning styles in adaptive hypermedia: Problems and solutions", in Proceedings of the WWW 2004, 2004, pp. 114-123.

[13] Sanders D (2008). Progress in machine intelligence. Ind ROB-an int journal 35 (6), pp 485-487. 
[14] Microsoft Corporation (2009) Asynchronous Pluggable Protocols [Online] Available:

\section{http://msdn.microsoft.com/workshop/networking/pluggable}

[15] R. Dunn and K. Dunn, Teaching students through their individual learning styles: A practical approach. Reston, VA: Reston Publishing, 1978.

[16] P. Honey and A. Mumford, The manual of learning styles. Maidenhead: Homey, 1986.

[17] R. M. Felder and L. K. Silverman, "Learning and teaching styles in engineering education”, Engineering Education, vol. 7, pp. 674-681, 1988.

[18] R.M.Felder and J Spurlin (2005) Applications, reliability and validity of the Index of Learning Styles. Jrnl of Eng Ed 21 (1), pp $103-112$.

[19] Litzinger, TA; Lee, SH; Wise, JC, et al. (2007). A psychometric study of the Index of Learning Styles. Jrnl of Eng Ed 96 (4), pp 309-319.

[20] R.M.Felder and R Brent (2005) Understanding student differences. Jrnl of Eng Ed 94 (1), pp 57 - 72.

[21] R. M.Felder and B. A. Soloman (2009) Index of Learning Styles Questionnaire [Online] Available: http://www.engr.ncsu.edu/learningstyles/ilsWeb.html

[22] Sanders DA, Urwin-Wright SD, Tewkesbury GE et al (2005). Pointer device for thin-film transistor and cathode ray tube computer screens. Electronics Letters 41 (16), pp 894-896.

[23] Chester S, Tewkesbury G and Sanders D et al (2007). New electronic multi-media assessment system. Web Information Systems and Technologies 1, pp 414-420.

[24] Sanders DA and Tewkesbury GE (2009). A pointer device for TFT display screens that determines position by detecting colours on the display using a colour sensor and an Artificial Neural Network. Displays 30 (2), pp 84-96.

[25] Sanders D (2008). Controlling the direction of "walkie" type forklifts and pallet jacks on sloping ground. Assembly Automation 28 (4), pp 317-324.

[26] Sanders DA and Baldwin, A (2001). X-by-wire technology. Total vehicle technology: challenging current thinking pp 3-12. 